\title{
New results in cosmology
}

\section{Subir Sarkar*}

Department of Physics, 1 Keble Road,_Oxford OX1 3NP, UK

E-mail: is.sarkar@physics.ox.ac.uks'

\begin{abstract}
From an observational perspective cosmology is today in excellent shape advances in instrumentation and data processing have enabled us to study the universe in detail back to when the first galaxies formed, map the fluctuations in the cosmic microwave background which provide a measure of the overall geometry, and reconstruct the thermal history reliably back to at least the primordial nucleosynthesis era. However recent deep studies of the Hubble expansion rate have suggested that the universe is accelerating, driven by some form of 'dark' (vacuum) energy. If true, this implies a new energy scale in Nature of $\mathcal{O}\left(10^{-3}\right) \mathrm{eV}$, well below any known scale of fundamental physics. This has refocussed attention on the notorious cosmological constant problem at the interface of general relativity and quantum field theory. It is possible that the resolution of this situation will require fundamental modifications to our ideas about gravity.
\end{abstract}

\section{Introduction}

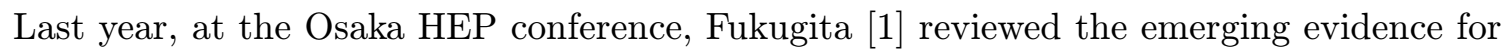
the possibility that the evolution of the universe today is being driven by a cosmological constant - Einstein's "greatest blunder" [2] $]$. In the intervening period there has been growing acceptance of this amazing claim by the astronomical community, and even by many particle physicists. If true, the implied small but non-zero energy density of the present vacuum poses a deep mystery for any fundamental theory [3i].

In the Standard Model Lagrangian, the term corresponding to the cosmological constant is one of the two 'super-renormalisable' terms allowed by the gauge symmetries, the second one being the quadratic divergence in the mass of fundamental scalar fields due to radiative corrections $\left[\begin{array}{l}4 \\ 4\end{array}\right]$. To tame the latter sufficiently in order to explain the experimental success of the Standard Model has required the introduction of a supersymmetry between bosonic and fermionic fields which is (softly) broken at about the Fermi scale. Thus the cutoff scale of the Standard Model, viewed as an effective field theory, can be lowered from the Planck scale, $M_{\mathrm{P}} \equiv\left(8 \pi G_{\mathrm{N}}\right)^{-1 / 2} \simeq 2.4 \times 10^{18} \mathrm{GeV}$, down to the

\footnotetext{
${ }^{*}$ Speaker.
} 
Fermi scale, $M_{\mathrm{EW}} \sim G_{\mathrm{F}}^{-1 / 2} \sim 300 \mathrm{GeV}$, albeit at the expense of introducing over 150 new masses and other parameters in the Lagrangian (as well as requiring delicate control of the many non-renormalisable operators which can generate flavour-changing neutral currents, nucleon decay etc, so as not to violate experimental bounds). This implies a minimum contribution to the vacuum energy density from quantum fluctuations of $\mathcal{O}\left(M_{\mathrm{EW}}^{4}\right)$, i.e. "halfway" down (on a logarithmic scale) from the Planck scale towards the energy scale of $\mathcal{O}\left(M_{\mathrm{EW}}^{2} / M_{\mathrm{P}}\right)$ corresponding to the observationally indicated vacuum energy. Thus even the introduction of supersymmetry cannot eradicate a discrepancy by at least a factor of $\sim 10^{60}$ between expectations and observations.

It is generally recognised that a true resolution of the cosmological constant problem can only be achieved in a full quantum theory of gravity. Recent developments in string theory and the possibility that there exist new dimensions in Nature have generated many interesting ideas concerning possible values of the cosmological constant [- However it remains true that there is no generally accepted solution to the problem of the discrepancy referred to above. Of course the cosmological constant problem is not new but there had always been the expectation that somehow we would understand one day why it is exactly zero. However if it is in fact non-zero and dynamically important today, the crisis is even more severe since it also raises a cosmic 'coincidence' problem, viz. why is present epoch of expansion special? The resolution of these conundrums may well require modifications to the construction of cosmological models (and the interpretation of astronomical data) in terms of Einstein's general relativity, although to date no such alternative which is phenomenologically satisfactory has been presented either.

Given this sorry situation on the theoretical front, I will focus in this talk solely on the new observational developments and in particular provide a critical assessment of the evidence for a cosmological constant. For lack of time I will not discuss the continuing theoretical activity in early universe cosmology concerning baryogenesis, inflation, particle dark matter etc. The most exciting development in this area has been the realisation that if there are new dimensions in Nature then they may have dramatically altered the evolution of the early universe so the standard lore [i6.] may have to be substantially rewritten. However attempts to constrain such new physics from cosmological arguments have also brought home the fact that we still have no detailed understanding of the early universe before the big bang nucleosynthesis $(\mathrm{BBN})$ era begining at $\sim 1$ s. In principle we know the physics underlying the evolution back to the epoch of electroweak symmetry breaking at $\sim 10^{-12} \mathrm{~s}$ but it appears that neither this event, nor the subsequent QCD confinement at $\sim 10^{-6} \mathrm{~s}$, would have left any detectable relics [i]. Thus early universe theorists presently have a free hand in speculating about the thermal history before the BBN era, in attempting to account for the known relics, viz. the baryon asymmetry, the abundance of dark matter and the density perturbation which seeded the growth of large-scale structure (LSS) and left its imprint on the cosmic microwave background (CMB). Hopefully the rapid observational progress being made on the cosmological front, particularly in precision studies of the CMB, will provide valuable constraints on (and possibly clues to) the physical processes that created our observable universe and all it contains. 


\section{The observational situation}

That we live in an universe which has been hotter in the past has recently been confirmed directly with the measurement of a higher temperature for the CMB at high redshift. This is done by observing fine-structure transitions between atomic levels of $\mathrm{C} I$ in quasar absorption systems (QAS) - cold gas clouds along the line of sight to distant quasars. Such measurements have already been possible for some time but the inferred temperatures have had to be considered as upper limits because alternative excitation mechanisms (i.e. other than being immersed in a Planckian radiation bath) may have contributed to the observed level populations. Recently an absolute CMB temperature measurement of $T_{\mathrm{CMB}}=10 \pm 4 \mathrm{~K}$ has been made in a QAS at redshift $z=2.338$ [i] such competing mechanisms through simultaneous measurements of the rotational levels in $\mathrm{H}_{2}$. (Subsequently a possible problem with this measurement has been noted but an even better constrained measurement of $T_{\mathrm{CMB}}=12.1_{-8.2}^{+1.7} \mathrm{~K}$ at $z=3.025$ has been reported

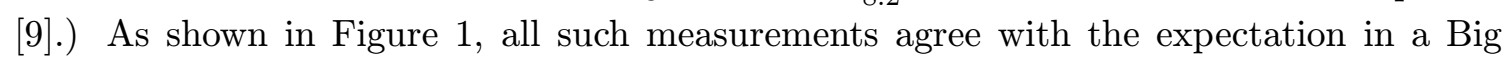
Bang cosmology that the blackbody temperature of the CMB increases with the redshift $\propto(1+z)$. This cannot be accomodated within the "Quasi-Steady State Cosmology" in which the CMB arises through thermalisation of starlight [1] ${ }_{1}^{1}[-1]$ - a mechanism that was already severely constrained by the closeness of the observed CMB spectrum to the Planck form [i] 1 in. The prospects for constraining other alternative cosmologies by further such measurements have been discussed in detail [i] $\left.{ }_{1}^{1} \overline{2}\right]$.

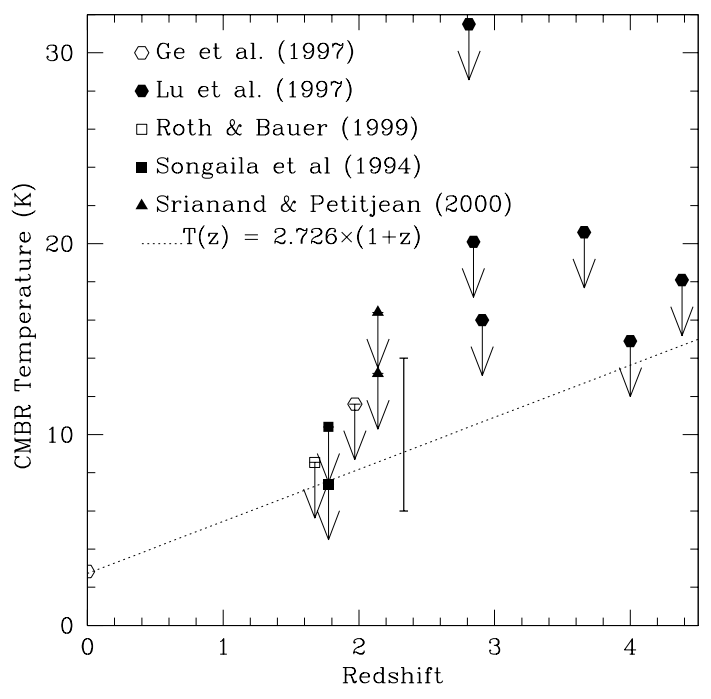

Figure 1: Measurements of the CMB temperature at various redshifts compared with the standard expectation (dotted line) normalised to the COBE measurement at $z=0$ (from Ref. [짐]).

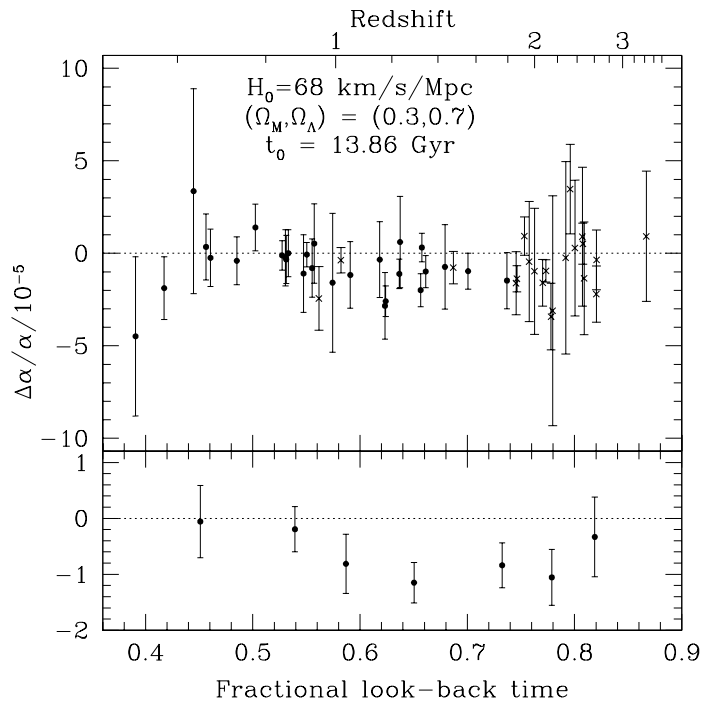

Figure 2: Inferred time variation in the finestructure constant from observations of QAS. The bottom panel shows an arbitrary binning of the raw results in the upper panel (from Ref. $\left[\overline{1} \overline{6}_{\mathbf{l}}^{\prime}\right)$.

For example such measurements also imply constraints on time-variations of fundamental constants, in particular of the fine-structure constant $\alpha$. This is a possibility that 


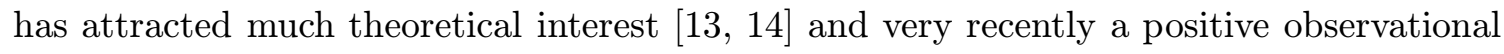
result has been claimed [i] $\underline{1}_{1}^{1}, \overline{1}_{1}^{1} \overline{6}_{i}^{i}$. Four independent samples were obtained (using three optical data sets and two $21 \mathrm{~cm}$ and mm absorption systems) spanning upto $\sim 90 \%$ of the age of the universe; each sample yields a smaller value of $\alpha$ in the past [i1 $\left.{ }^{1}{ }_{1}^{1}\right]$. As shown in Figure $\overline{2}$, the optical sample of 49 QAS shows a $4 \sigma$ deviation: $\Delta \alpha / \alpha=(-0.72 \pm 0.18) \times 10^{-5}$ over the redshift range $z \sim 0.5-3.5$. Moreover it is argued that correcting for any possible systematic effects would increase the significance of the deviation of $\Delta \alpha / \alpha$ from zero [i] $\left.\bar{i}_{i}\right]$. If true this is a most exciting result since it implies the existence (in 4-dim effective field theory) of a very weakly coupled ultralight scalar field which would mediate a 'fifth force'

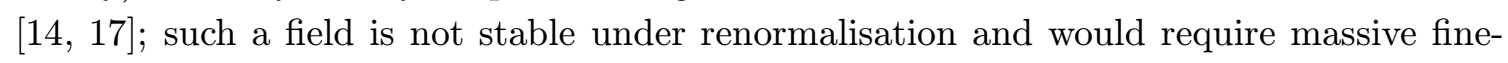
tuning, further exacerbating the cosmological constant problem [1 $1 \overline{2} \bar{l}]$. However studies of the $21 \mathrm{~cm}$ line of hydrogen yield a conflicting bound at $z=1.6$ of $\Delta \alpha / \alpha=(3.5 \pm 5.5) \times 10^{-6}$

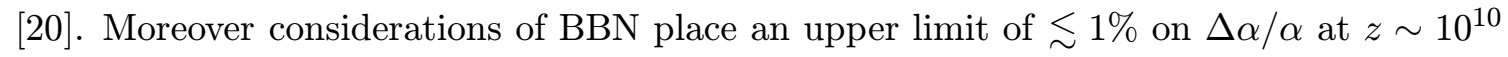

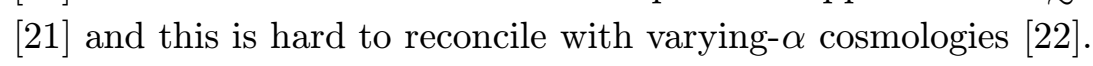

\subsection{The age of the universe}

Returning to the standard cosmology, another recent observational highlight has been the direct measurement of the age of an extremely metal-poor (i.e. very old) star in the halo of our Galaxy through detection of the $385.957 \mathrm{~nm}$ line of singly ionized ${ }^{238} \mathrm{U}$, as shown

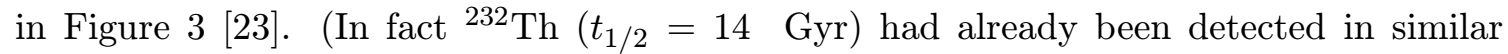
stars and used to infer an age of $15.6 \pm 4.6 \mathrm{Gyr}$ [24] but it decays so little over the lifetime of the Universe that ${ }^{238} \mathrm{U}\left(t_{1 / 2}=4.5 \mathrm{Gyr}\right)$ is in principle a more sensitive probe. $)$ The derived abundance, $\log (\mathrm{U} / \mathrm{H})=-13.7 \pm 0.14 \pm 0.12$ corresponds to an age of $12.5 \pm 3 \mathrm{Gyr}$, consistent with the (recently revised) age of $11.5 \pm 1.3 \mathrm{Gyr}$ for the oldest stars in globular clusters inferred from stellar evolution arguments [2] $\left.{ }_{2} \overline{1}\right]$. Although concerns remain about the internal consistency of this result, e.g. the observed Th/Eu ratio implies a much smaller age for this star [2] $\overline{4}]$, direct radioactive dating avoids many of the uncertainties that have plagued estimates of globular cluster ages using the "Main Sequence Turn-off Method", e.g. the inferred values had to be revised downward by several Gyr following the Hipparcos calibration of stellar distances obtained by parallax measurements [2 $\left.\overline{2}_{\overline{5}} \bar{i}\right]$. Other methods which determine the distance and the age simultaneously (e.g. using the "Luminosity Function", see Figure $\underset{4}{\overline{4}}$ ) have been developed and yield a minimum age for globular clusters of 10.5 Gyr $\left[\overline{2} \overline{\sigma_{i}}\right]$. To obtain the age of the universe we must add $\sim 0.2-2 \mathrm{Gyr}$, the estimated epoch of galaxy/star formation, to the above number.

\subsection{The Hubble constant}

The age thus obtained must be consistent with the expansion age of the universe, derived from measurements of the present Hubble expansion rate. This year the Hubble Space Telescope Key Project has presented its final results on $H_{0}$ [2근] . Based on direct measurements of the distances to 18 nearby spiral galaxies (using Cepheid variables) and using these to calibrate five secondary methods, they find that all data are consistent with $H_{0}=72 \pm 3 \pm 7 \mathrm{~km} \mathrm{~s}^{-1} \mathrm{Mpc}^{-1}$, as shown in Figure $H_{0}$ are obtained by 'physical' methods e.g. measurements of time delays in gravitationally 


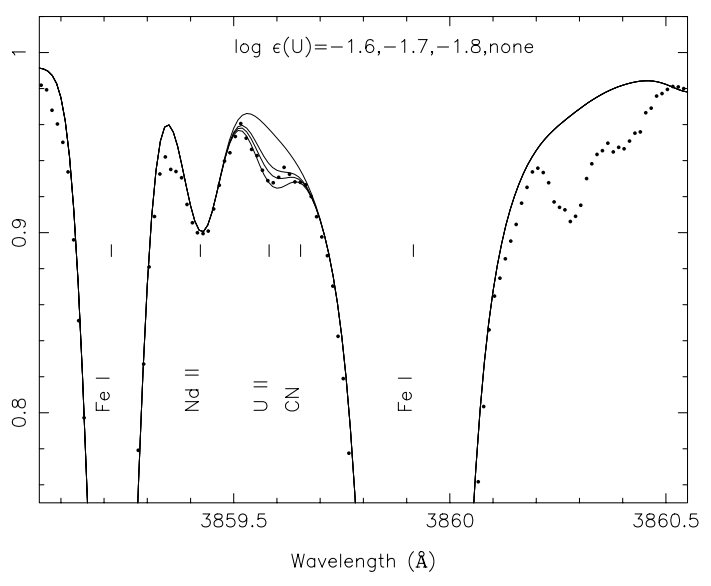

Figure 3: Detection of ${ }^{238} \mathrm{U}$ in the old halo star CS31802-001. Synthetic spectra for three assumed values of the abundance are compared with the data (from Ref. [2] $\left.{ }_{2}^{2} 3_{1}\right]$ ).

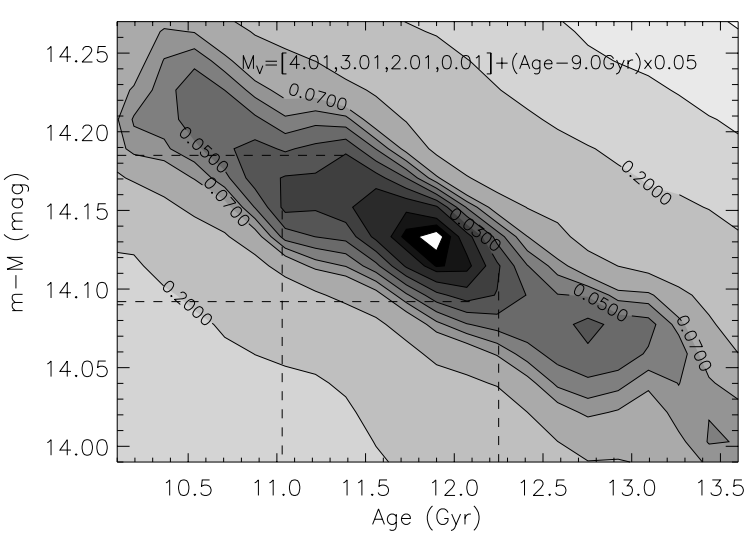

Figure 4: Simultaneous determination of the distance modulus and age for the globular cluster M55 by the "Luminosity Function" method (from Ref. [2] $[2]$ ).

lensed systems, and the Sunyaev-Zeldovich effect (SZE) in X-ray emitting galaxy clusters, which bypass the traditional 'distance ladder' and probe far deeper distances than the objects used by the Key Project. At present ten multiply-imaged quasars have measured time delays or are being monitored - the two best constrained systems (PG1115+080 and B1608+656) yield $H_{0}=61 \pm 11 \mathrm{~km} \mathrm{~s}^{-1} \mathrm{Mpc}^{-1}$ using a non-parametric modelling of the lenses [" $\overline{2} \overline{2} \overline{8}]$, while similar low values are found (with larger possible lens modelling uncer-

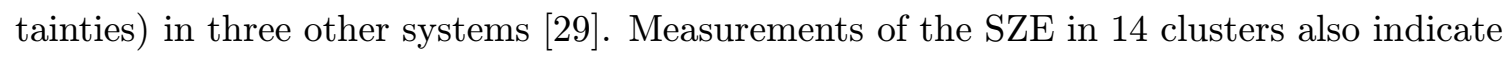
a value of $H_{0} \sim 60 \mathrm{~km} \mathrm{~s}^{-1} \mathrm{Mpc}^{-1}$ with prsently a large $(\sim 30 \%)$ systematic uncertainty [i] $\left.\overline{3}_{0}\right]$. Rowan-Robinson has argued that the Key Project data need to be corrected for local peculiar motions using a more sophisticated flow model than was actually used, and also for metallicity effects on the Cepheid calibration - this lowers the value of $H_{0}$ inferred from the same dataset to $63 \pm 6 \mathrm{~km} \mathrm{~s}^{-1} \mathrm{Mpc}^{-1}[\underline{3} \overline{3} \overline{1}]$.

It is often stated that an Einstein-DeSitter (E-deS) universe with $\Omega_{\mathrm{m}}=1, \Omega_{\Lambda}=0$ is too short-lived to be compatible with measurements of $H_{0}$ and $t_{0} \cdot{ }^{1}$ As seen in Figure ${ }^{6} \overline{6}^{1}$, adopting the Key Project measurement of the present expansion rate and a reasonable age for the universe does rule out this model at the $2 \sigma$ level and favours an open universe with $\Omega_{\mathrm{m}} \lesssim 0.3$ or a flat universe with a cosmological constant. However in view of the continuing debate concerning the value of $H_{0}$, this conclusion cannot as yet be considered definitive.

\subsection{The deceleration parameter}

The most exciting observational developments have undoubtedly been in measurements of the deceleration parameter $q \equiv \mathrm{d} H^{-1} / \mathrm{d} t-1$, which equals 0.5 for the E-deS model where $H \propto t^{2 / 3}$, and -1 for a DeSitter (deS) model with $\Omega_{\mathrm{m}}=0, \Omega_{\Lambda}=1$ which has $H$ constant.

\footnotetext{
${ }^{1}$ Here $\Omega_{\mathrm{m}}$ and $\Omega_{\Lambda}$ are the energy densities of matter and the vacuum in units of the critical density $\rho_{\mathrm{c}} \equiv 3 H_{0}^{2} / 8 \pi G_{\mathrm{N}} \simeq\left(3 \times 10^{-3} \mathrm{eV}\right)^{4}$, i.e. $\Omega_{m}+\Omega_{\Lambda}=1-\kappa$ where the curvature term $\kappa$ is 0 for a flat universe.
} 


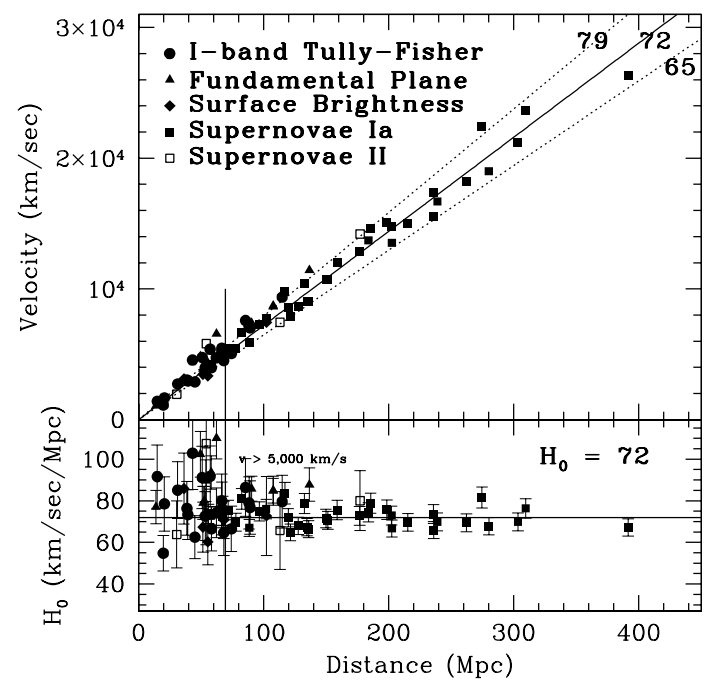

Figure 5: Hubble diagram for Cepheidcalibrated secondary distance indicators; the bottom panel illustrates the decrease in fluctuations (due to peculiar velocities) with increasing distance (from Ref. [י-27,
$\mathrm{H}_{0}$ and $t_{0}$ Measurements to $\pm 10 \%$

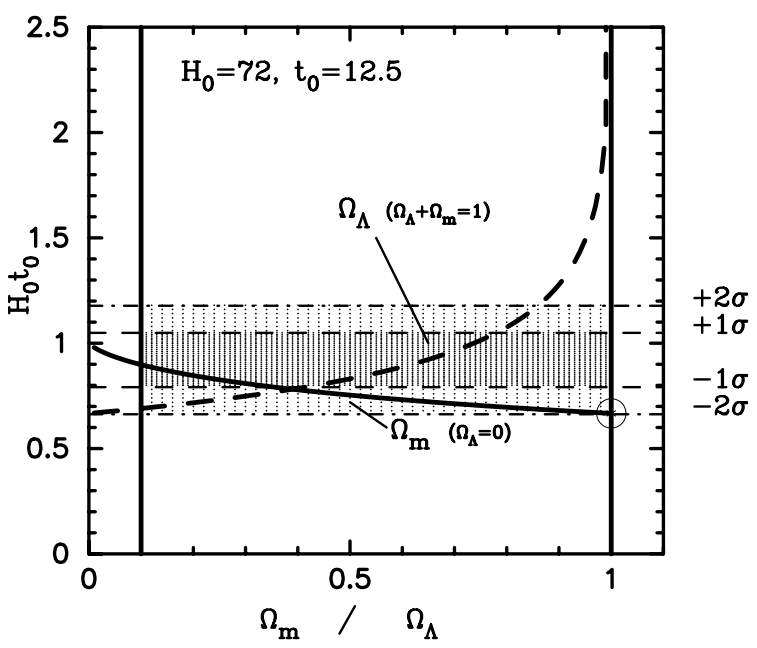

Figure 6: The time-scale problem for an E-deS universe (marked with a circle), assuming $\pm 10 \%$ uncertainties for the indicated values of the present expansion rate and age (from Ref.[2메)

This has been done through impressive deep studies of the Hubble diagram of Type Ia

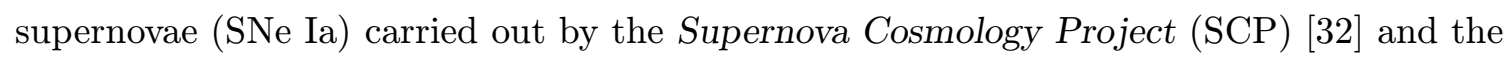
High-z SN Search Team (HZT) [i] $\left.\overline{3}_{3}\right]$. Their basic observation is that distant supernovae (upto $z \sim 1$ corresponding to looking back $\sim 10 \mathrm{Gyr}$ ) are upto $\Delta m \sim 0.5$ mag (corresponding to $10^{\Delta m / 2.5}-1 \simeq 60 \%$ ) fainter than would be expected for a decelerating universe such as the E-deS model. This has been interpreted as implying that the expansion rate has been speeding up since then, thus the observed SNe Ia are actually further away than expected. ${ }^{2}$ The obvious possibility that the SNe Ia appear fainter because of absorption by intervening dust can be constrained since this would also lead to reddening (unless the dust has unusual properties [35i] $)$. It is more difficult to rule out the possibility of evolution, i.e. that the distant SNe Ia are intrinsically fainter. Many careful analyses have been made of these possibilities by the observing teams themselves as well as by others. A detailed

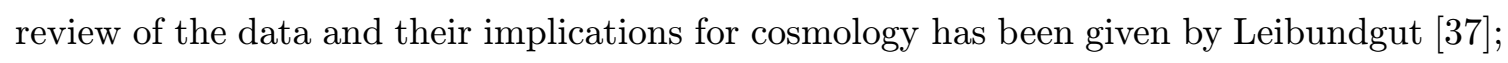
the summary of the present situation below draws largely on this work.

Briefly, SNe Ia are observationally known to be a rather homogeneous class of objects, with intrinsic peak luminosity variations $\lesssim 20 \%$, hence particularly well suited for cosmological tests which require a 'standard candle' [3] $\left.{ }^{2} \bar{n}\right]$. They are characterised by the absence of hydrogen in their spectra [i] $\left.{ }^{3} \overline{9}\right]$ and are believed to result from the thermonuclear explosion of a white dwarf, although there is as yet no "standard model" for the progenitor(s) $\left[\_\overline{4} \overline{0}\right]$. However it is known (using nearby objects with independently known distances) that

\footnotetext{
${ }^{2}$ The measured apparent magnitude $m$ of a source of known absolute magnitude $M$ yields the 'luminosity distance': $m-M=5 \log \left(\frac{d_{\mathrm{L}}}{\mathrm{Mpc}}\right)+25$, where $d_{\mathrm{L}}=(1+z) \int_{0}^{z} \frac{\mathrm{d} z^{\prime}}{H\left(z^{\prime}\right)}$ is sensitive to the expansion history [i $\underline{3}_{\underline{1}}$.
} 
the time evolution of SNe Ia is tightly correlated with their peak luminosities such that the intrinsically brighter ones fade faster - this can be used to make corrections to reduce the scatter in the Hubble diagram using various empirical methods, viz. the $\Delta m_{15}$ template

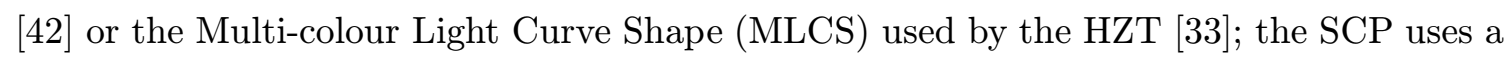
simple 'stretch factor' to normalise the observe apparent peak magnitudes [322]. It should be emphasised that such corrections are essential to reduce the scatter in the data sufficiently so as to allow significant cosmological deductions. It is therefore a matter for concern that, as seen in Figure $\overline{\mathbf{z}}_{1}$, the different methods make magnitude corrections that do not compare well with each other $\left[\overline{3} \overline{3}_{1}, \overline{3} \overline{3}_{2} \bar{Z}_{1}\right]$ - as they should if indeed some intrinsic physical property of SNe Ia was responsible for the observed correlations. Moreover as shown in Figure $\bar{q}$, distant SNe Ia appear to be bluer in colur than nearby ones; this suggests that the derived reddening may have been underestimated [i] $\overline{3} \bar{z}]$. A recent reanalysis of the data also finds that both teams have underestimated the effects of host galaxy extinction and that the peak luminosity-decay time correlation is much weakened if SNe Ia not observed before maximum light are excluded; this further weakens the significance of the claimed

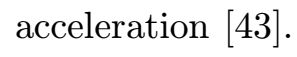

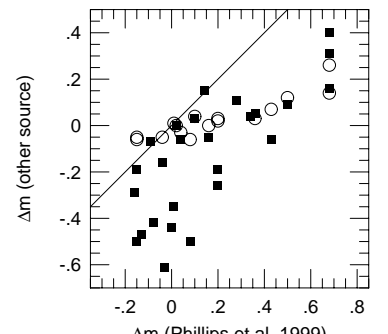

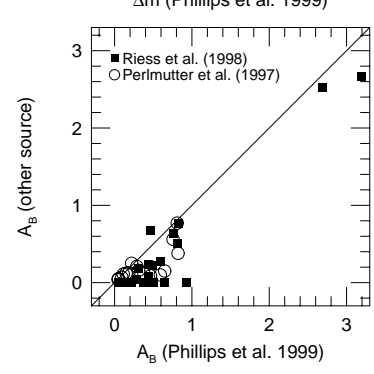

Figure 7: Comparison of the magnitude corrections (top panel) and of the inferred absorption (bottom panel) for the nearby SNe Ia sample, for the three different methods used to analyse the data (from Ref. [4 $\left.4 \overline{1}_{-}\right]$).

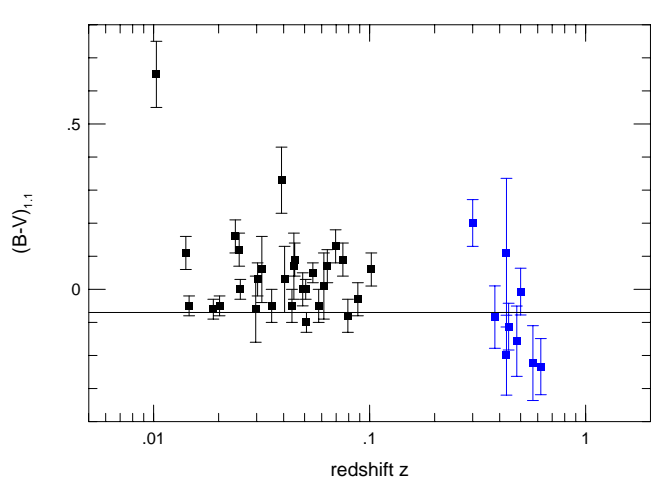

Figure 8: The observed colours of low redshift [42 2 vs high redshift [33 Ref. [3년]

Figure ${ }_{1-1}^{-1}$ shows the magnitude-redshift diagram of SNe Ia obtained by the two teams. Averaging over the distant supernovae, it is found that they are $0.20 \pm 0.06$ magnitudes fainter at $z \sim 0.5$ than in an (empty) universe expanding at constant rate when analysed using the $\Delta m_{15}$ correction method; this decreases to $0.14 \pm 0.06$ using the MLCS method

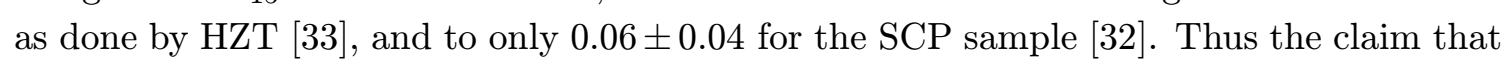
cosmic acceleration has been observed is no more significant than the recent indications for the Higgs at LEP! The best fit ' $\Lambda$ CDM model' - a low matter density, cosmological 
constant dominated flat universe with $\Omega_{\mathrm{m}} \simeq 0.3, \Omega_{\Lambda} \simeq 0.7$ - is however favoured by measurements of the age and present expansion rate as discussed earlier, and also by independent measurements of the matter density and spatial curvature to be discussed, so has become the new "standard cosmological model" for the astronomical community.

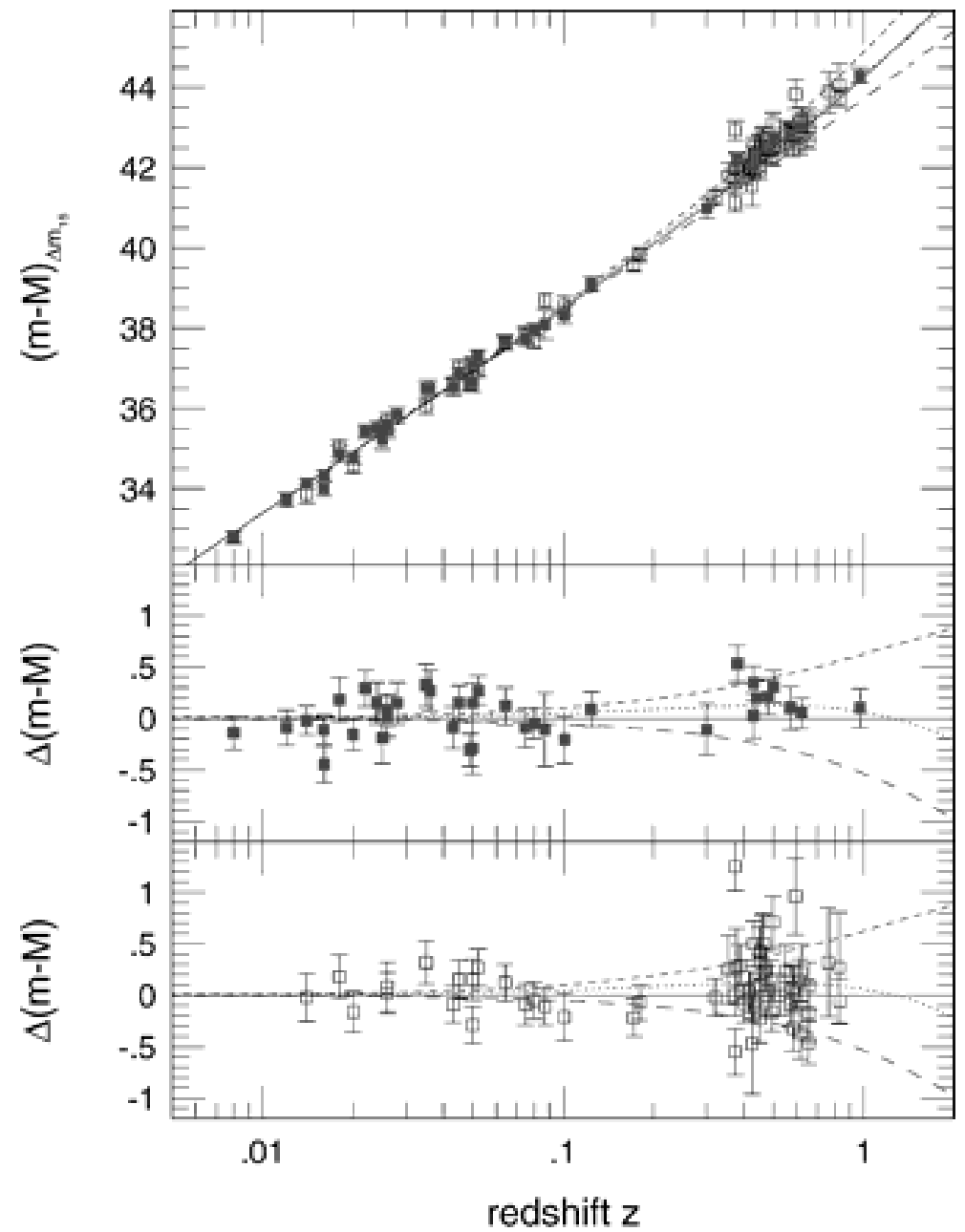

Figure 9: The Hubble diagram of SNe Ia (top panel) compared with the expectation for an empty universe (full line), an E-deS universe $\Omega_{\mathrm{m}}=1, \Omega_{\Lambda}=0$ (long-dashed line), a DeS universe $\Omega_{\mathrm{m}}=0, \Omega_{\Lambda}=1$ (dashed line), and a flat $\Omega_{\mathrm{m}}=0.3, \Omega_{\Lambda}=7$ universe (dotted line), with all data normalised to the $\Delta m_{15}$ method [42in. The lowest panel shows separately the Supernova Cosmology

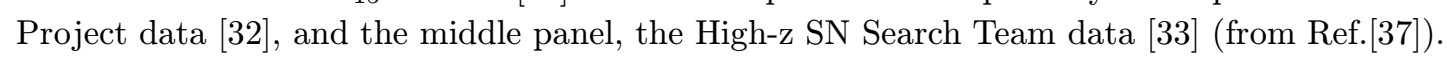

More recently the serendipitous discovery of a very distant supernova (SN1997ff) in the Hubble Deep Field at a redshift $z \sim 1.7$ is claimed to have largely eliminated alternative explanations such as absorption by "grey" dust or luminosity evolution in favour of a cosmological constant [4] [4]. This is because for $z \gtrsim 1$ the cosmological constant becomes unimportant relative to the increasing matter density $\left(\propto(1+z)^{3}\right)$ so the expansion should be seen to be slowing down at such epochs; the transition from acceleration to deceleration occurs at $z=\left(2 \Omega_{\Lambda} / \Omega_{m}\right)^{1 / 3}-1$. It is argued [i-4īi] that SN1997ff is indeed a SNe Ia because 
the host galaxy is an elliptical rather than a spiral and this identification is consistent with the observed colours and time evolution. As seen in Figure $1 \overline{0} \overline{0}_{n}^{\prime}$ the inferred luminosity then does indicate deceleration in that SN1997ff is brighter than would be expected for an uniform rate of expansion; this argues against absorption by dust or simple forms of luminosity evolution as being responsible for the curvature in the Hubble diagram at lower redshifts. However it has been noted that SN1997ff may have been significantly brightened by gravitational lensing due to two foreground elliptical galaxies which are close to the

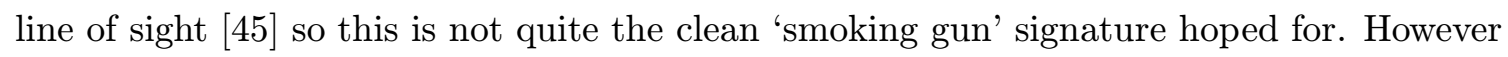
it does illustrate the effectiveness of such deep observations. The proposed Supernova Acceleration Probe (SNAP) space mission [4흠] will observe $\sim 2000$ SNe Ia out to a redshift of $z \simeq 1.2$ and the increase in statistics and improved control of systematics should be able to improve the accuracy of $\Omega_{\mathrm{m}}$ and $\Omega_{\Lambda}$ determinations to a few per cent! Only through such future measurements can the claim of recent cosmic acceleration, driven by vacuum energy, be definitively tested.

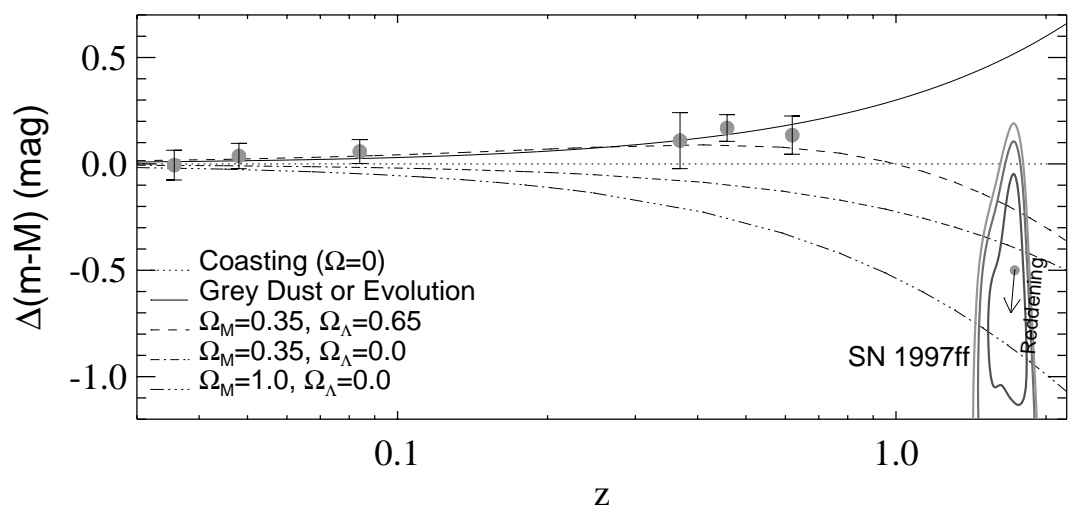

Figure 10: Hubble diagram of SNe Ia normalised to an empty uniformly expanding universe with redshift binned data from the SCP and HZT for redshifts $\lesssim 1$ and the data for SN1997ff at $z \sim 1.7$ (from Ref. [4י[4]

\section{The spatial curvature and the matter density}

Even though the direct evidence for a non-zero $\Omega_{\Lambda}$ is rather weak as argued above, many astronomers have nevertheless accepted the findings. This is really because of two independent observations which also suggest, that there is a substantial cosmological constant.

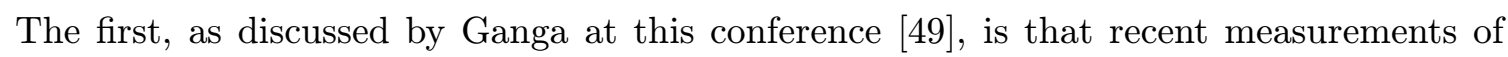
degree-scale angular fluctuations in the CMB provide a measurement of the sound horizon (a 'standard rod') at last scattering [i] $\kappa \simeq 0$ i.e. the universe is spatially flat $[\overline{5} \overline{\overline{0}}]$. The second is that, as recognised for some time, several types of observations suggest that the amount of matter which participates

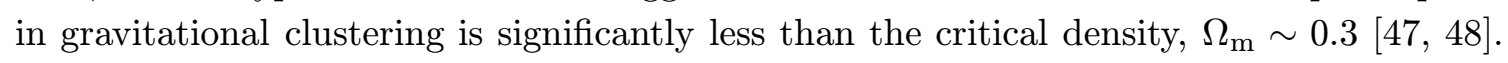
By the 'cosmic sum rule' this then requires that there be some form of 'dark energy', unclustered on the largest spatial scales probed in the measurements of $\Omega_{\mathrm{m}}$, with an energy density of $1-\Omega_{\mathrm{m}} \sim 0.7$. This is indeed consistent with the value of $\Omega_{\Lambda} \sim 0.7$ suggested by 
the SNe Ia data leading to the widespread identification of the dark energy with vacuum energy. ${ }^{3}$ However it should be emphasised that this is rather indirect evidence for a cosmological constant and there may be other explanations for the apparent shorfall between the matter density and the critical density required by the CMB. In view of the importance of the conclusion, each line of evidence ought to be critically judged on its own merits.

For example the position of the first acoustic peak in the angular power spectrum of the CMB implies that the spatial curvature is close to zero only if the primordial scalar density perturbation, presumably generated during inflation, is assumed to be adiabatic and gaussian. This is indeed what is expected in simple inflationary models [" $[2 \overline{6} \overline{6}]$ but given that we have no "standard model" of inflation it is legitimate to consider more general perturbations, e.g. including isocurvature modes. The most general cosmological perturbation in an universe filled with photons, baryons, neutrinos, and cold dark matter (CDM)

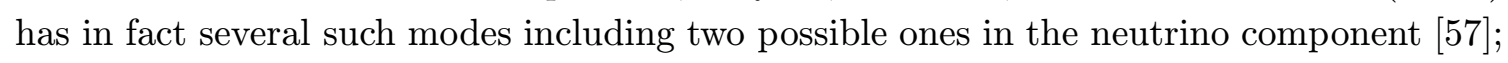
moreover the adiabatic and isocurvature modes are in general correlated. If such modes exist then the inference from the position of the first peak that the universe is flat is severely compromised [61]. Even if we assume that the universe is flat, the extracted values of other cosmological parameters can be significantly altered if there are isocurvature modes

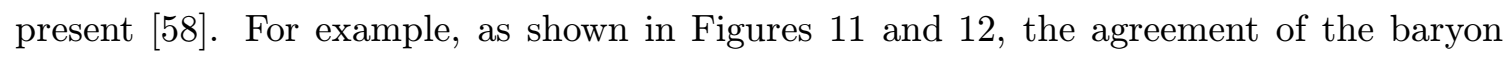
density inferred from the CMB data with the value indicated by BBN [5은, the perturbation is adiabatic. Of course it is not clear what physical mechanism can excite e.g. neutrino isocurvature perturbations after neutrinos decouple at $\sim 1 \mathrm{MeV}$, nevertheless this illustrates that "precision" determinations of cosmological parameters from the CMB cannot yet be considered fully robust. To distinguish experimentally between isocurvature and adiabatic perturbations requires measurements of the CMB polarisation which will be

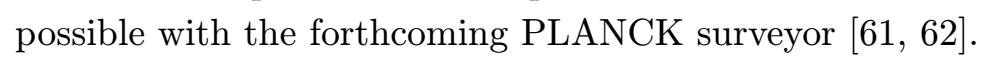

Another important property of the primordial scalar density perturbation about which we have no direct knowledge is its spectral shape. It has been common practice to deduce cosmological parameters from analyses of LSS and CMB data, assuming that the primordial perturbation has a scale-invariant 'Harrison-Zeldovich' form with power-law index $n=1$. Indeed many of the arguments for a low matter density universe (e.g. the 'shape factor' of the LSS power spectrum) are crucially dependent on this assumption; even allowing e.g. a small 'tilt' $n \simeq 0.9$ for the spectrum allows a high matter density universe to be consistent with the data [63i]. Moreover such a departure from scale-invariance is indeed expected in models of 'new inflation' [" $[\overline{5} \overline{6} \overline{6}]$ ] and explicit models based on supergravity which do yield the required tilt have been presented [i $[\overline{4} \overline{4}]$. It is interesting to note that consistency of

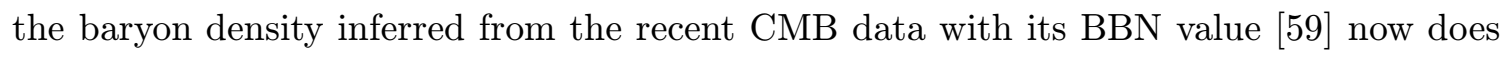

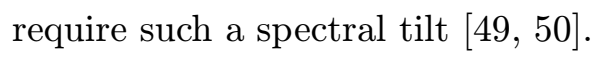

\footnotetext{
${ }^{3}$ In seeking to understand why the vaccum energy has begun to dominate at the present epoch many theorists have pursued the idea that it may be the energy of an evolving scalar field — 'quintessence' $\left[52^{1},{ }_{1}^{\prime}, 53\right.$ ' In order to be affected by the Hubble expansion such a field must have a mass of $\mathcal{O}\left(H_{0}\right) \sim 10^{-33} \mathrm{eV}$, much smaller than the height of its potential, $\sim 3 \times 10^{-3} \mathrm{eV}$, thus raising formidable difficulties in relating it to fundamental theory. However if the dark energy is parameterised in terms of its equation of state $w \equiv p / \rho$, the SNe Ia observations are in fact best fitted by $w \simeq-1\left[\begin{array}{c}5 \\ -1\end{array}\right.$
} 


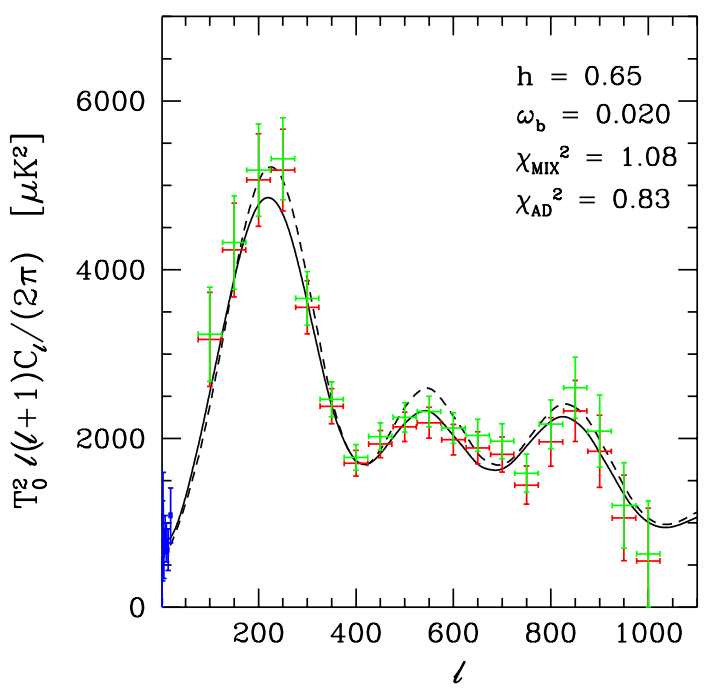

Figure 11: Fits to the CMB data assuming purely adiabatic perturbations (dashed line) and mixed perturbations with $12 \%$ isocurvature content (solid line), adopting the baryon density $\omega_{\mathrm{b}}=\Omega_{\mathrm{b}} h^{2}=0.02$ indicated by BBN.

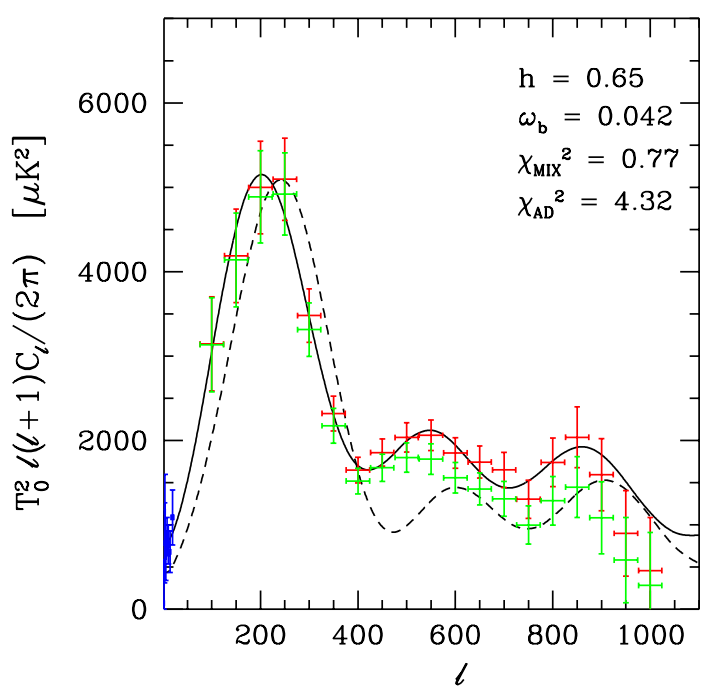

Figure 12: The same but now for an assumed baryon density twice the $\mathrm{BBN}$ value; the adiabatic case is now a poor fit but the mixed case (with $69 \%$ isocurvature compo-

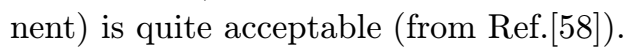

In fact the primordial perturbation may not even be scale-free; indeed it is sensitive to the dynamics of inflation (and the behaviour of fields other than the inflaton itself) and is likely to have features such as 'steps' and 'bumps' [6흠. Of course there is no unique prediction in this regard so it is quite appropriate that analyses of CMB and LSS data should still assume a scale-free spectrum (but now allowing a 'tilt' for consistency with the data). However the inferred cosmological parameters can change substantially if we allow for a more complex primordial spectrum, e.g. a 'step' in the spectrum permits a

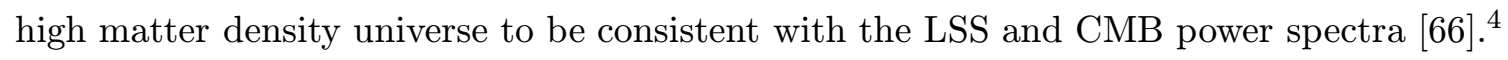
The only independent way to constrain such possibilities is through more detailed analyses of structure formation, to test the important assumption of a scale-free (and gaussian) primordial perturbation.

There has indeed been impressive recent progress in mapping the present day distri-

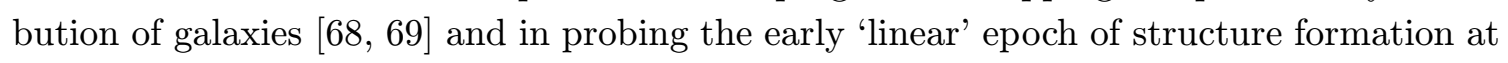
higher redshifts through studies of the "Lyman- $\alpha$ forest" in QAS [i] $70 \overline{0}]$. It has also become possible to probe the dark matter distribution directly through studies of the 'shear' in-

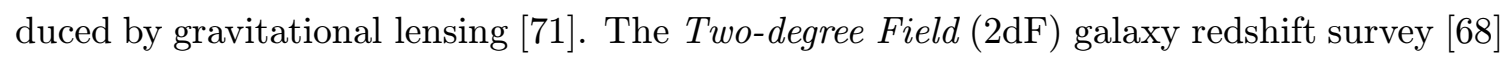
has detected the expected distortion in redshift-space due to peculiar (i.e. non-Hubble) velocities induced by the gravitational collapse of structure [6 $\left.68^{2}\right]$. If galaxies do trace the dark matter distribution, as is indicated by studies of high-order correlation functions in the same data, then this does provide direct evidence for a low density universe with

\footnotetext{
${ }^{4}$ The CMB data used (from the Boomerang experiment) has subsequently been revised but our conclusion still holds (e.g. using the latest combined data set $\left[{ }_{15}^{-} 0_{0}\right]$ ), although an $\Omega_{\mathrm{m}}=1$ universe no longer gives a good fit as before. In view of the uncertainties in the measurements, particularly at high multipoles, it

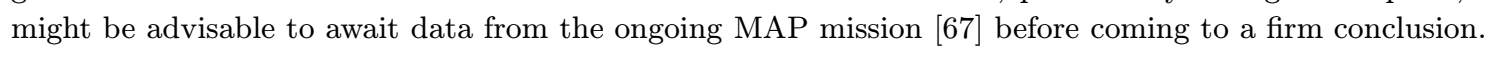


$\Omega_{m}=0.27 \pm 0.06$ on the largest scales (upto several hundred Mpc) probed so far. This is also consistent with the measured power spectrum. By doing a joint fit to the 2DF and CMB data (assuming a scale-free primordial spectrum of adiabatic fluctuations), evidence is found for a cosmological constant with $0.65<\Omega_{\Lambda}<0.85$, [i]2i] it should soon be possible to test the robustness of such results with even fewer assumed 'priors', in particular the nature of the primordial density perturbation, and address other concerns about structure formation [푸국.

\section{Conclusions}

Thus for the moment there is a 'cosmic concordance' model with $\Omega_{\mathrm{m}} \sim 0.3, \Omega_{\Lambda} \sim 0.7$ which is consistent with all astronomical data but has no explanation in terms of fundamental physics. One might hope to eventually find explanations for the dark matter (and baryonic) content of the universe in the context of physics beyond the Standard Model but there appears to be little prospect of doing so for the apparently dominant component of the universe - the cosmological constant. Cosmology has in the past been a data-starved science so it has been appropriate to consider only the simplest possible cosmological models in the framework of general relativity. However now that we are faced with this serious confrontation between particle physics and cosmology, it is perhaps time to even consider possible alternatives to general relativity.

This is of course not trivial - general relativity has been extensively tested on (relatively small) astronomical scales [i] $\left.{ }_{i} \overline{1}\right]$ and the standard cosmology based on it certainly gives

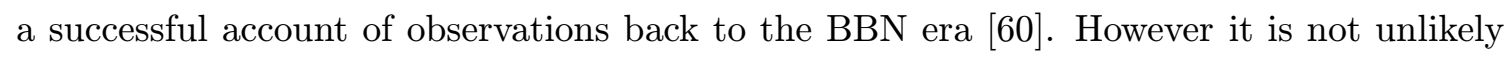
that the ferment of current theoretical ideas concerning 'brane-world' might suggest small modifications to our notions about gravity, even sacred texts like the Equivalence Principle, which turn out to be significant in the cosmological context [7 $7 \overline{3}$ ]. Of course astronomers are entitled to, and will continue to, analyse their data in terms of well-established physics. But it is important for it to be recognised that the cosmological constant is not just another parameter among many specifying a cosmological model.

Landu famously said "Cosmologists are often wrong, but never in doubt". The situation today is perhaps better captured by Pauli's enigmatic remark - the present interpretation of the data may be "... not even wrong" However we are certainly not without doubt! Moreover the crisis posed by the recent astronomical observations is not one that confronts cosmology alone; it is the spectre that haunts any attempt to unite two of the most successful creations in physics - quantum field theory and general relativity. The future of cosmology is inextricably intertwined with that of fundamental physics and promises to be most exciting indeed.

\section{Acknowledgments}

I am grateful to Bruno Leibundgut and Michael Rowan-Robinson for very helpful correspondance concerning the measurement of cosmological parameters. I would also like to thank the organisers of this excellent conference for the invitation to present this review. 


\section{References}

[1] M. Fukugita, in XXXth International Conference on High Energy Physics, Osaka, World-Scientific, 2000, Vol.1, p.299, [hep-ph/0012214].

[2] For a historical perspective, see, N. Straumann, in Third International Conference On Dark Matter In Astro And Particle Physics, World-Scientific, 2001, p.110, [astro-ph/0009386i.

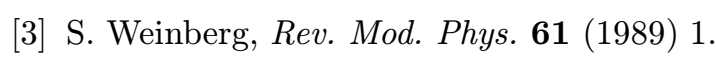

[4] see, e.g., F. Zwirner, in International Europhysics Conference on High Energy Physics, Brussels, World-Scientific, 1996, p.943, [hep-ph/9601300].

[5] For recent reviews, see, e.g. E. Witten, [hep-ph/0002297];

S. Weinberg, [astro-ph/0005265i];

M. Dine, [hep-th/0107259i].

[6] E.W. Kolb and M.S. Turner, The Early Universe, Addison-Wesley, 1990.

[7] see, e.g., M. Laine, [hep-ph/011 1349].

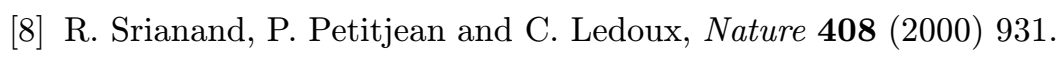

[9] P. Molaro, S.A. Levshakov, M. Dessauges-Zavadsky and S. D'Odorico, lastro-ph/0111589̄].

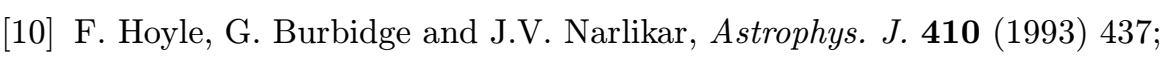
For a recent update, see, G. Burbidge, [astro-ph/0108051].

[11] P.J.E. Peebles, D.N. Schramm, E.L. Turner and R.G. Kron, Nature 352 $\overline{1} 1991 \overline{1} 76 \overline{9}_{1}^{\prime}$

[12] J.M. LoSecco, G.J. Mathews and Y. Wang,

[13] see, e.g., W.J. Marciano, 'Phys. Rev. Lett. $\mathbf{5 2}(1984) 489$ '

C. Hill and G.G. Ross, iNucl. Phys. B 311 (1988) 253;

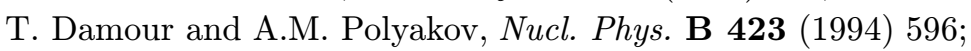

J.D. Barrow and J. Magueijo, 'Astrophys. J. $\mathbf{5 3 2}(2000)$ L87.'.

[14] S.M. Carroll, 'Phys. Rev. Lett. 81 (1998) 3067.

[15] J. K. Webb et al., 'Phys. Rev. Lett. 87.

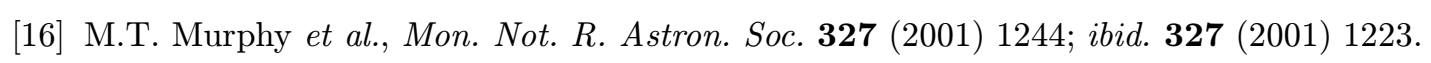

[17] G. Dvali and M. Zaldarriaga, [hep-ph/0108217].

[18] T. Banks, M. Dine and M.R. Douglas, [hep-ph/0112059].

[19] X. Calmet and H. Fritzsch, [hep-ph/0112110].

[20] L.L. Cowie and A. Songaila, Asstrophys.J.453 (1995) 596 ; see also C.L. Carilli et al., 'Phys.Rev. Lett. 85 (2000) 5511 ,

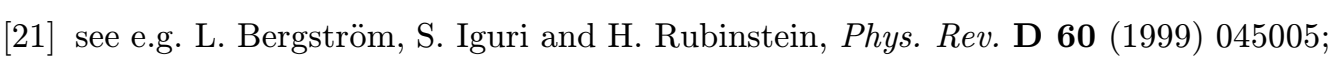

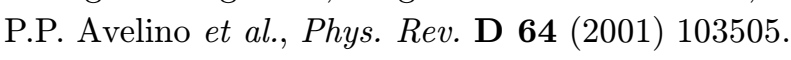

[22] D. Kabat and A. Rajaraman, Phys. Lett. B $\mathbf{5} \mathbf{1} \mathbf{6}-2001 \overline{3} 33^{\prime}$

[23] R. Cayrel et al., 'Nature $\mathbf{4} \mathbf{0} \mathbf{9}(2001) 69 \overline{1}$.'

[24] J.W. Truran, S. Burles, J.J. Cowan and C. Sneden, [astro-ph/0109526].

[25] B. Chaboyer, Phys. Rept. 307 $(19 \overline{9} \overline{8}) 2 \overline{3}$. 
[26] R. Jimenez, in Dark Matter in Astrophysics \& Particle Physics 1998, Adam Hilger, 1999, p.170, [astro-ph/9810311].

[27] W.L. Freedman et al., 'Ástrophys. J. $5533(2001)+47_{1}^{\prime}$

[28] L.L.R. Williams and P. Saha, 1 Astron. $\bar{J}$. 119 $(2000) 43 \overline{9}$.

[29] L.V.E. Koopmans and C.D. Fassnacht,

[30] J.E. Carlstrom et al., [astro-ph/0103480].

[31] M. Rowan-Robinson, in Third International Conference on Identification of Dark Matter, World Scientific, 2001, [astro-ph/0012026].

[32] S. Perlmutter et al., iAstrophys.

[33] A.G. Riess et al., 'A Astron. J. $116 \mathbf{1}(1998) 1009$;

[34] S. Carroll, W. Press and E.L. Turner, Ann. Rev. Astron. Astrophys. 30 (1992) 499.

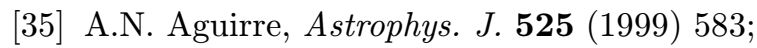

A.N. Aguirre and Z. Haimann, ibid. $\mathbf{5 3 2}(2000) 28$.

[36] P.S. Drell, T.J. Loredo and I. Wasserman, 'Astrophys. J. 530 (2000) 593.

[37] B. Leibundgut, Ann. Rev. Astron. Es Astrophys. 39 (2001) 67 .

[38] D. Branch, 'Ann. Rev. Astron. É Astrophys. $\mathbf{3} 6$ (1998) 17.

[39] A.V. Fillipenko, iAnn. Rev. Astron. ${ }^{2}$ Astrophys. 35 (1997) 309 .

[40] W. Hillebrandt and J.C. Niemeyer,

[41] B. Leibundgut, 'Âstron. Astrophys. Rev. 10 (2000) $17 \overline{9}$.

[42] M.M. Phillips et al., 'Astron. J. 118 (1999) 1766.'.

[43] M. Rowan-Robinson, preprint (2001), submitted to Mon. Not. R. Astron. Soc.

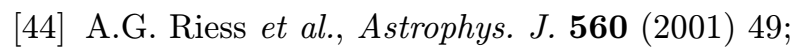
see also M.S. Turner and A.G. Riess, [astro-ph/0106051i.

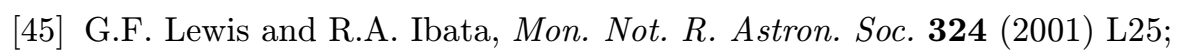

E. Mörtsell, C. Gunnarsson and A. Goobar, Astrophys. J. 561 (2001) 106.

[46] SNAP (URL: http://snap.lbl.gov).

[47] P.J.E. Peebles, [astro-ph/0102327i].

[48] see e.g. M. Davis, Phys. Rept. $33 \overline{3}(2000) 147$;

N.A. Bahcall, J.P. Ostriker, S. Perlmutter and P.J. Steinhardt, ŚScience $\mathbf{2} 8 \mathbf{4}(1999) 1481$.

[49] K. Ganga, in EPS International Conference on High Energy Physics, Budapest, 2001 (D. Horvath, P. Levai, A. Patkos, eds.), JHEP (http://jhep.sissa.it/) Proceedings Section, PrHEP-hep2001/283.

[50] For a comprehensive analysis of all CMB experiments, see, X. Wang, M. Tegmark and M. Zaldariagga, [astro-ph/0105091].

[51] see e.g. W. Hu and S. Dodelson, [astro-ph/0110414];

R. Dürrer, [astro-ph/0109522]. 
[52] P.J.E. Peebles and B. Ratra, 'AAstrophys.

R.R. Caldwell, R. Dave and P.J. Steinhardt, Phys. Rev. Lett. 80 (1998) 1582!

[53] For reviews, see, S. Carroll, Liv. Rev. Rel. $4(2001)$ 1, lestro-ph/0004075i ;

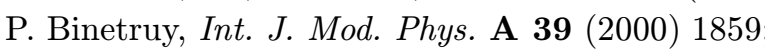

V. Sahni and A. Starobinsky, IInt. J. Mod. Phys. D 9 $(20 \overline{0} 0 \overline{0}) \overline{3} 73$

[54] S. Perlmutter, M.S. Turner and M. White, Phys. Rev. Lett. 83 (1999) $6 \overline{7}_{1}^{\prime}$

[55] T.D. Saini, S. Raychaudhury, V. Sahni and A.A. Starobinsky, 'Phys. Rev. Lett. 85 $\mathbf{2}(2000)$ i. - - 1162

[56] See, e.g. A.D. Linde, Particle Physics and Inflationary Cosmology, Harwood Academic Press, 1990 ;

A. Liddle and D.H. Lyth, Cosmological Inflation and Large-Scale Structure, Cambridge University Press, 2000.

[57] M. Bucher, K. Moodley and N. Turok, ${ }_{2}$ hys. Rev. D'62 $(2000) 083508$

[58] R. Trotta, A. Riazuelo and R. Dürer, 'LPhys. Rev. Lett. $8 \overline{7}(\mathbf{2} 001) 231301$ '

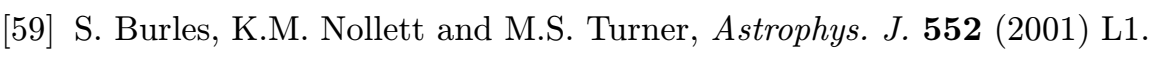

[60] For a more conservative assessment, see, B.D. Fields and S. Sarkar, 2001 WWW Review of particle Physics (URL: http://pdg.lbl.gov/).

[61] M. Bucher, K. Moodley and N. Turok, 'P hyss. Rev. Lett. 87. (2001) 191301;

[62] K. Enqvist and H. Kurku-Suonio, 'P

[63] see, e.g. M. White, D. Scott and J. Silk, Science 268 (1995) $829 ;$

[64] G.G. Ross and S. Sarkar, iNucl. P Phys. B $461-19 \overline{9}) \overline{5} \overline{9}$;

J.A. Adams, G.G. Ross and S. Sarkar, Phys.Lett. B B91 (1997) 271'

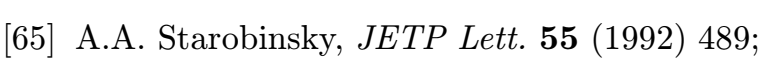

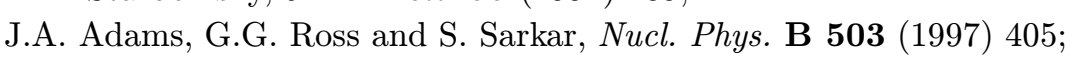

D. Chung, E.E. Kolb, A. Riotto and I.I. Tkachev, 1 Phys.Rev. D 62

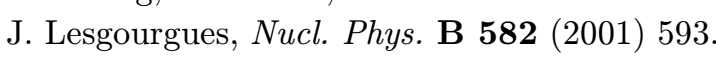

[66] J. Barriga, E. Gaztanaga, M. Santos and S. Sarkar, 'Mon. Not. R. Astron. Soc. 324 (2001)i -

[67] (URL: http://map.gsfc.nasa.gov/).

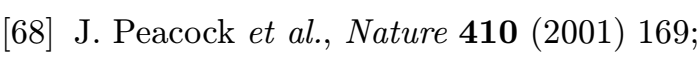

W. Percival et al., ['astro-ph/0105252i;

L. Verde et al., [astro-ph/011216i].

[69] I. Zehavi et al., [astro-ph/0106470];

S. Dodelson et al., lastro-ph/0107421].

[70] R.A.C. Croft, D.H. Weinberg, M. Pettini, L. Hernquist and N. Katz, Astrophys. (1999) 1 ;

M. Zaldariagga, L. Hui and M. Tegmark, Astrophys. J. $557 .(2001) 519 !$

[71] Y. Mellier et al., [astro-ph/0101130i].

[72] G. Efstathiou et al., [astro-ph/0109152].

[73] see, e.g., T. Damour, 'Eur. Phys. J. 15 (2000) 121; lgr-qc/0109063]. 